\title{
Can patients reliably identify safe, high quality care?
}

\author{
Sarah E. Tevis, Ryan K. Schmocker, Gregory D. Kennedy \\ Department of Surgery, University of Wisconsin, Wisconsin, United States
}

Correspondence: Sarah Tevis. Address: 600 Highland Avenue, Madison, WI 53792, United States. E-mail: stevis@uwhealth.org

Accepted: June 24, 2014

Online Published: July 3, 2014

DOI : 10.5430/jha.v3n5p150

URL: http://dx.doi.org/10.5430/jha.v3n5p150

\section{Abstract}

The Hospital Consumer Assessment of Healthcare Providers and Systems (HCAHPS) survey is a publicly reported tool that measures patient satisfaction. As both patients and Centers for Medicare \& Medicaid Services (CMS) reimbursement rely on survey results as a metric of quality of care, we reviewed the current literature to determine if patient satisfaction correlates with quality, safety, or patient outcomes. We found varying associations between safety culture, process of care measure compliance, and patient outcomes with patient satisfaction on the HCAHPS survey. Some studies found inverse relationships between quality and safety metrics and patient satisfaction. The measure that most reliably correlated with high patient satisfaction was low readmission rate. Future studies using patient specific data are needed to better identify which factors most influence patient satisfaction and to determine if patient satisfaction is a marker of safer and better quality care. Furthermore, the HCAHPS survey should continue to undergo evaluations to assure it generates predictable results.

\section{Key words}

Patient satisfaction, Safety, Quality of care

\section{I ntroduction}

The Centers for Medicare \& Medicaid Services (CMS) and the Agency for Healthcare Research and Quality (AHRQ) aligned in 2002 to develop a standardized, publicly reported survey of patient reported hospital care. The survey was entitled the Hospital Consumer Assessment of Healthcare Providers and Systems (HCAHPS). The three main objectives of the HCAHPS survey were: (1) to allow objective comparisons across hospitals for consumers, (2) to motivate hospitals to improve quality of care, and (3) to increase transparency of care through public reporting of survey results ${ }^{[1]}$. The development and implementation of the HCAHPS survey is outlined in Figure 1. Over the course of three years, the survey was developed, tested in a three state test pilot, revised based on psychometric properties, formatted for public reporting, and endorsed by the National Quality Forum ${ }^{[1-4]}$. The final survey consists of 32 questions and is administered to adult patients discharged from a medical, surgical or obstetric service ${ }^{[1,2]}$. In 2006, hospitals were invited to use the survey and two years later the Inpatient Prospective Payment System (IPPS) required participating hospitals to collect HCAHPS surveys and report the results publicly ${ }^{[1,3]}$.

The HCAHPS survey has been validated after rigorous testing to ensure that survey results are objective and comparable across hospitals. Hospital level reliability was found to range from 0.66-0.89 (median $=0.88$ ) and internal consistency 
reliabilities ranged from $0.51-0.88$ (median $=0.72)^{[5]}$. Survey results are adjusted for mode of administration and patient case mix, after which adjusted scores are reported on the Hospital Compare website quarterly ${ }^{[1,3,6-11]}$. After adjusting for patient case mix variables (patient reported health status, education, age, primary language, service line, hospital response rate and service-age interaction), the HCAHPS Survey has been shown to have satisfactory internal consistency reliability and hospital-level reliability ${ }^{[5,12,13]}$. Therefore, the ability to make valid patient experience comparisons across hospitals, the first goal of developing the HCAHPS survey, has been achieved. The third goal, to publically report survey results, was achieved in March 2008. CMS reports survey results on the Hospital Compare website quarterly (http://www.medicare.gov/hospitalcompare/search.html).

Figure 1. The development and implementation of the HCAHPS survey

HCAHPS: Hospital Consumer Assessment of Healthcare Providers and Systems

CMS: Centers for Medicare \& Medicaid Services

AHRQ: Agency for Healthcare Research and Quality

IPPS: Inpatient Prospective Payment System

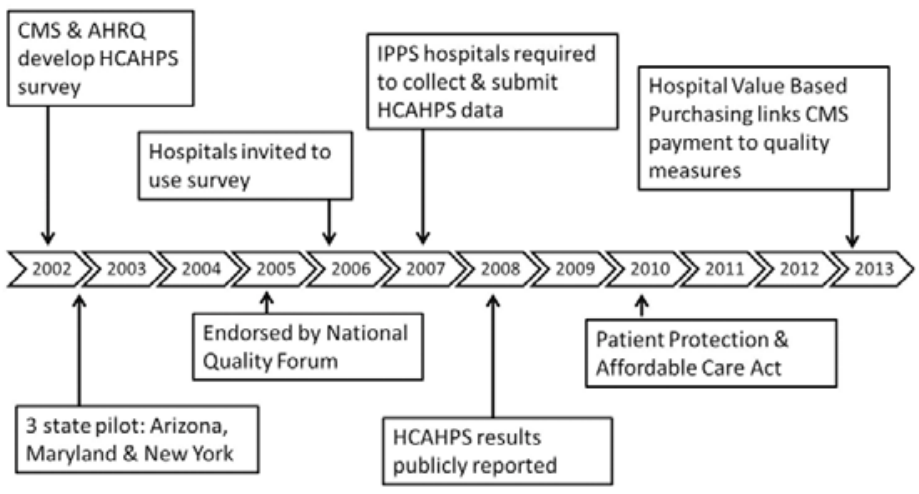

Both by instituting a new payment structure and by publicly reporting patients' perceptions of care, Medicare has incentivized low performing hospitals to improve and high performing hospitals to maintain HCAHPS scores. Initially, CMS implemented a pay-for-reporting model, which tied public reporting of HCAHPS results to Medicare reimbursement through the IPPS. Payments to IPPS hospitals are based on diagnosis related groups (DRGs) and adjusted for hospital factors, such as location and teaching status, and patient variables, such as proportion of low income patients ${ }^{[3]}$. Recently, the Patient Protection and Affordable Care Act has tied IPPS hospitals' reimbursement to the Hospital Value-Based Purchasing (Hospital VBP) Total Performance Score (TPS). The Hospital VBP program consists of a clinical process of care domain, which comprises $70 \%$ of the TPS, and the patient experience of care domain, which makes up $30 \%$ of the TPS. The Hospital VBP program TPS consists of 25 measures including 8 HCAHPS measures. The included HCAHPS domains are: Communication with nurses, Communication with doctors, Staff responsiveness, Pain management, Communication about medications, Discharge information, Cleanliness and quietness, and Overall rating of hospital. Currently $1 \%$ of Medicare reimbursement is dependent on TPS performance, with plans to increase to 2\% by 2017. As HCAHPS results account for the Patient Experience Domain, or 30\% of the TPS, there is a strong monetary incentive for hospitals to improve patient perception of care ${ }^{[14,15]}$.

Despite a push to improve patients' perceptions of care, it remains unclear that the second goal of the HCAHPS survey, to motivate hospitals to improve quality of care, has been sufficiently addressed. To reflect this goal, the literature regarding the development and implementation of the survey often uses the phrases "patient satisfaction" and "quality of care" interchangeably. While patients' perceptions of care are an important component of the quality of care hospitals provide, we sought to evaluate whether patient satisfaction on the HCAHPS survey correlates with traditional measures of safe, high quality care. We hypothesized that patient satisfaction on the HCAHPS survey would not correlate with quality measures, safety culture, and patient outcomes.

\section{Methods}

We performed a review of the literature in PubMed for articles published between January 1, 2000 and January 1, 2014. Searches were limited to the English language. Search terms for the outcome of interest, patient satisfaction on the HCAHPS survey, included: HCAHPS, CAHPS, perception of care, and patient satisfaction. Each of these search terms 
was paired with terms to represent the three areas of interest: quality of care, safety culture, and patient outcomes including: outcomes, quality, safety, and process measures. An initial review yielded 36 abstracts and articles. An ancestral search identified 37 more publications for a total of 73 articles after removal of duplicates. After compiling the articles into a single list, a single reviewer assessed studies for exclusion criteria. Four studies assessed patient satisfaction on surveys other than HCAHPS, seven papers were opinion articles, 29 papers were limited to the development of the survey, and 23 papers focused on patient and hospital factors. We were left with 10 pertinent articles after excluding articles that did not address the relationship between scores on the HCAHPS survey and quality/safety outcomes.

\section{Results}

\subsection{I ncluded studies}

We identified 10 studies that met the inclusion criteria of this study. A flowchart of eligible studies and reasons for exclusion is displayed in Figure 2. Publication dates ranged from 2001-2013. Articles were divided into three categories: culture of safety, process measure compliance, and patient outcomes. All articles are summarized below.

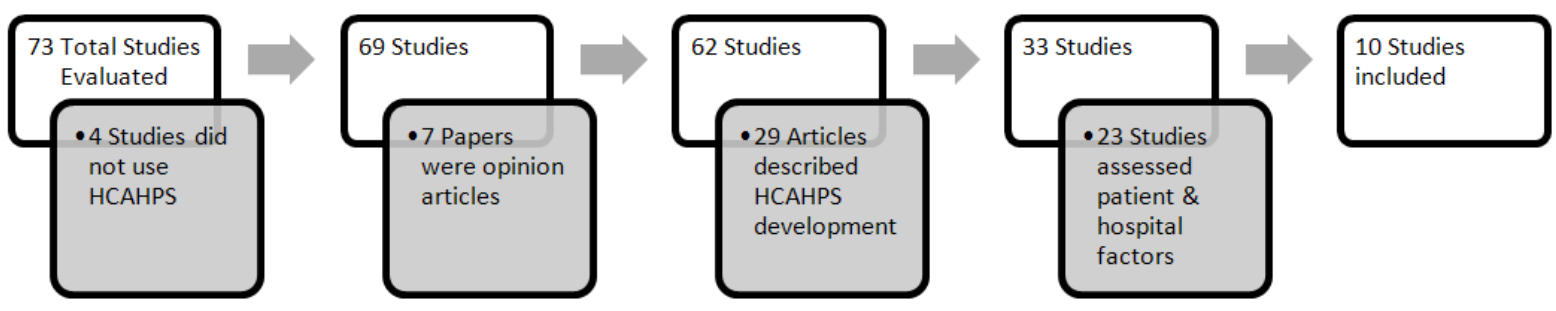

Figure 2. The criteria used to identify eligible studies

\subsection{Culture of safety}

Establishing a culture of safety in health care organizations has been found to empower employees to identify and report dangerous situations while building trust throughout the organization ${ }^{[16]}$. Furthermore, improvements in perception of safety climate have been found to be associated with improved patient outcomes and a decline in adverse events ${ }^{\text {[17-19] }}$. Numerous tools exist to monitor attitudes and culture of safety in hospitals, many of which were derived from other high-risk industries such as aviation. However, only two validated surveys are commonly used to assess hospital safety culture: the Safety Attitudes Questionnaire (SAQ) ${ }^{[20]}$ and the Hospital Survey on Patient Safety Culture (HSOPS) survey ${ }^{[21]}$. The SAQ was derived from a Flight Management Attitudes Questionnaire, which measured airline crew attitudes about the interpersonal aspects of crew performance. The questionnaire is 60 items, takes 10-15 minutes to complete, and has been demonstrated to have acceptable scale reliability $(0.9)^{[20]}$. The HSOPS survey was developed to assess safety climate and culture. The survey has 42 items asking respondents about patient safety issues, event reporting, and the culture for their work area. Reliability for composites of the HSOPS survey range from 0.62-0.85 (mean = 0.77) ${ }^{[21]}$. Both the SAQ and HSOPS survey measure teamwork, safety, working conditions, and perceptions of hospital management.

Our review identified two studies that analyzed safety culture and attitudes in relation to patient satisfaction on the HCAHPS survey (see Table 1). Lyu and colleagues ${ }^{[22]}$ compared SAQ results in surgery departments at 31 US hospitals with overall satisfaction scores. The authors found no association between overall safety culture scores and patient satisfaction ( $p=.07$ ), although higher scores on the following domains ( 2 of 6) were found to correlate with higher satisfaction scores: teamwork climate $(p=.01)$ and safety climate $(p=.03)$. Interestingly, stress recognition, the measure of hospital employees' ability to recognize a decline in performance related to stressors such as increased work load, fatigue, and emergency situations, was found to have a significant inverse relationship with patient satisfaction $(p=$ $.008)^{[22]}$. 
Table 1. Safety culture and patient satisfaction

\begin{tabular}{|c|c|c|c|c|}
\hline Author & Journal & Safety Survey & Satisfaction measure & Associations $^{*}$ \\
\hline Lyu $^{* *}$ & $\begin{array}{l}\text { JAMA Surg } \\
2013\end{array}$ & SAQ & $\begin{array}{l}\text { Overall Satisfaction } \\
\text { Ranking of } 9 \text { or } 10 \\
\text { Recommendation of hospital }\end{array}$ & $\begin{array}{l}\text { Teamwork Climate }(+) \\
\text { Safety Climate }(+) \\
\text { Stress recognition }(-)\end{array}$ \\
\hline Sorra & $\begin{array}{l}\text { J Patient Saf } \\
2012\end{array}$ & HSOPS & $\begin{array}{l}\text { Composite HCAHPS score } \\
\text { (average of all domains) }\end{array}$ & $\begin{array}{l}\text { Error Communication }(+) \\
\text { Nonpunitive response to error }(+) \\
\text { Organizational learning }(+) \\
\text { Perception of patient safety }(+) \\
\text { Staffing }(+) \\
\text { Supervisor expectations }(+) \\
\text { Teamwork within units }(+) \\
\text { Patient Safety Grade }(+) \\
\text { HSOPS Composite }(+)\end{array}$ \\
\hline
\end{tabular}

Note. ${ }^{*}(+)$ indicates a positive correlation between the survey domain and patient satisfaction, (-) indicates a negative correlation between the survey domain and patient satisfaction; ${ }^{* *}$ Notably, no association was identified between overall safety culture scores and patient satisfaction ( $\left.p=.07\right)$.

SAQ: Safety Attitudes Questionnaire, HSOPS: Hospital Survey on Patient Safety Culture, HCAHPS: Hospital Consumer Assessment of Healthcare Providers and Systems

A separate study by Sorra et al. ${ }^{[23]}$ examined the relationship between HSOPS survey performance and HCAHPS scores and found results that contradicted the Lyu study. In this study, an analysis of 73 hospitals found a positive correlation between the majority (9 of 15) of HSOPS measures and a composite HCAHPS score calculated by averaging the percentage of positive scores for all domains. The individual HCAHPS domains found to be significantly associated with the most HSOPS measures (12 of 15 each) included: communication with nurses, communication about medications, responsiveness of hospital staff, and hospital environment.

These conflicting results may be a product of the fact the studies examined the results of different surveys. Although both surveys have been found to have acceptable and similar reliability and predictive validity, previous analysis found that scores cannot be converted between the two surveys making direct comparisons difficult ${ }^{[24]}$. Furthermore, the two groups used different statistical techniques to control for bias in their studies. For example, the Lyu HSOPS study controlled for hospital size and ownership, whereas the Sorra SAQ study did not control for these factors. Given these conflicting methods, it is unclear whether safety attitudes and culture are related to patient satisfaction. Future studies should assess the relationship between culture of safety and patient satisfaction across multiple surveys to determine if there is a relationship and if it is consistent across survey types.

\subsection{Process measure compliance}

Process of care measures gauge hospital compliance with evidence based standards of care. The results are publically reported and performance on select measures is tied to CMS reimbursement as part of the Hospital VBP TPS. Two established measures are used to evaluate process measure compliance. The first, the Healthcare Effectiveness Data and Information Set (HEDIS) ${ }^{[25]}$ evaluates a wide range of clinical processes of care using administrative data paired with chart review and surveys. Second, the Hospital Quality Alliance (HQA) process measures ${ }^{[26]}$ assess compliance with standards of care for three medical conditions: acute myocardial infection (AMI), heart failure (HF), and pneumonia (PN). HQA process measures also exist for surgical patients, with monitoring of perioperative process measure compliance through the Surgical Care Improvement Project (SCIP) ${ }^{[26]}$.

Studies assessing process measure compliance in relation to patient satisfaction are listed in Table 2. Schneider and colleagues ${ }^{[27]}$ assessed patient satisfaction in association with the following HEDIS measures: primary and secondary prevention (mammography screening, eye exams for diabetic patients, beta blocker after myocardial infarction, cholesterol testing after cardiovascular event) and mental health care (30 day follow up after hospitalization and 
continuation of antidepressants). Patient satisfaction with getting needed care and health plan information were significantly associated with all HEDIS measures $(p<.05)$ except continuation of antidepressant medications. Hospital plans that were described as providing care quickly were associated with high compliance with mammography screening $(p=.0001)$ and follow up after hospitalization for mental illness $(p=.004)$. Satisfaction with physician communication was only associated with one HEDIS measure, mammography screening $(p=.02)$. In a regression analysis, the factors independently associated with HEDIS measures were satisfaction with health plan information and customer service, which significantly predicted 4 of 6 HEDIS measures.

Table 2. Process measure compliance and patient satisfaction

\begin{tabular}{|c|c|c|c|c|}
\hline Author & Journal & Process Measure & Satisfaction measure & Associations* \\
\hline Schneider & $\begin{array}{l}\text { Med Care } \\
2001\end{array}$ & HEDIS & $\begin{array}{l}\text { Getting needed care } \\
\text { Getting care quickly } \\
\text { Communication with doctors } \\
\text { Helpfulness of staff } \\
\text { Health plan information } \\
\text { Customer service }\end{array}$ & $\begin{array}{l}\text { Health plan information }(+) \\
\text { Customer service }(+)\end{array}$ \\
\hline Jha & $\begin{array}{l}\text { N Engl J Med } \\
2008\end{array}$ & HQA & $\begin{array}{l}\text { Overall satisfaction } \\
\text { Ranking of } 9 \text { or } 10\end{array}$ & $\begin{array}{l}\mathrm{AMI}(+) \\
\mathrm{CHF}(+) \\
\mathrm{PN}(+) \\
\operatorname{SCIP}(+)\end{array}$ \\
\hline Isaac & $\begin{array}{l}\text { Health Serv Res } \\
2010\end{array}$ & HQA & $\begin{array}{l}\text { Overall satisfaction } \\
\text { Ranking of } 9 \text { or } 10 \\
\text { Recommendation of hospital } \\
\text { Physician communication } \\
\text { Nurse communication } \\
\text { Medication communication } \\
\text { Pain management } \\
\text { Clean and quiet room } \\
\text { Staff responsiveness } \\
\text { Discharge information }\end{array}$ & $\begin{array}{l}\mathrm{AMI}(+) \\
\mathrm{CHF}(+) \\
\mathrm{PN}(+) \\
\operatorname{SCIP}(+)\end{array}$ \\
\hline Lehrman & $\begin{array}{l}\text { Med Care } \\
2010\end{array}$ & $\begin{array}{l}\text { HQA summary score } \\
\text { (average of all domains) }\end{array}$ & $\begin{array}{l}\text { HCAHPS summary score (average } \\
\text { of all domains) }\end{array}$ & HQA summary score $(+)$ \\
\hline Lyu & $\begin{array}{l}\text { JAMA Surg } \\
2013\end{array}$ & SCIP & $\begin{array}{l}\text { Overall satisfaction } \\
\text { Ranking of } 9 \text { or } 10 \\
\text { Recommendation of hospital }\end{array}$ & None \\
\hline
\end{tabular}

Note. ${ }^{*}(+)$ indicates a positive correlation between the survey domain and patient satisfaction, (-) indicates a negative correlation between the survey domain and patient satisfaction.

HQA: Hospital Quality Alliance, AMI: Acute myocardial infarction, CHF: Congestive heart failure, PN: Pneumonia, SCIP: Surgical Care Improvement Project, HEDIS: Healthcare Effectiveness Data and Information Set

Across studies, associations between HQA process measures and patient satisfaction have varied from strong positive associations to inverse correlations in the literature. A study of 2,429 US hospitals evaluated AMI, HF, PN, and SCIP HQA process measures in association with overall satisfaction (ranking of 9-10) on the HCAHPS survey ${ }^{\text {[28] }}$. After adjusting for hospital characteristics, better compliance with all four HQA process measures was significantly associated with improved overall patient satisfaction $(p<.001)$.

Isaac and colleagues ${ }^{[29]}$ obtained similar results in a study of 927 hospitals where HQA process measure compliance was compared with all HCAHPS measures. Better performance on pneumonia process measures was significantly associated with all HCAHPS measures, while compliance with surgical process measures (8 of 9) and acute myocardial infarction (7 of 9) measures also significantly correlated with patient satisfaction. Alternatively, congestive heart failure measures were only found to correlate with the 2 overall satisfaction measures (overall hospital rating and recommendation of hospital). 
A different approach was taken in a study by Lehrman et al. ${ }^{[30]}$ wherein they evaluated top performers in both summary scores for 22 HQA process measures and 8 HCAHPS measures in 2,583 hospitals. Process measure summary scores correlated positively with HCAHPS summary scores at the hospital level $(p<.0001)$. Interestingly, only $8 \%$ of hospitals scored in the top quartile for both the process measure score and the HCAHPS score. An additional $34 \%$ of hospitals scored in the top quartile for one of the two summary measures.

Lyu et al. ${ }^{[22]}$ evaluated SCIP process compliance in association with overall patient satisfaction across 31 hospitals. Evaluated measures included: outpatient and inpatient antibiotic prophylaxis, hair removal, urinary catheter removal within 48 hours following surgery, and deep vein thrombosis prophylaxis. Overall satisfaction was measured by the proportion of patients who ranked the hospital 9 or 10 out of 10, in addition to the percentage of patients who would definitely recommend the hospital. In contrast with previously published work, the study found no significant associations between SCIP compliance and overall patient satisfaction.

The majority of studies evaluating process measure compliance related to patient satisfaction found an association between these measures. Interestingly, the most recent study by Lyu and colleagues ${ }^{[22]}$ demonstrated no relationship between compliance with SCIP measures and patient satisfaction. This study assessed a much smaller number of hospitals by comparison and the conflicting results may be due to sampling bias or small sample size. Alternatively, these differences may be explained by changes over time or the use of surgical process measures as opposed to all available measures. Another explanation may be that any differences are underpowered as SCIP compliance is approaching $100 \%$ at many institutions. These combined results suggest that performance on the process of care measures is somewhat associated with patient satisfaction, but these associations are not universal.

\subsection{Patient outcomes}

The goal of developing a culture of safety and increasing compliance with process measures is ultimately to improve patient outcomes. Therefore, an important marker of quality and safety of patient care is patient outcomes. The most commonly tracked and reported outcomes include mortality, complications, and readmissions. These outcomes are monitored over varying time periods and with inconsistent criteria across medical and surgical specialties and available databases.

Table 3 lists five articles, which assess patient outcomes in relation to patient satisfaction. Elliott et al. ${ }^{[31]}$ evaluated patient satisfaction at the end of life as a way to better understand patient satisfaction and mortality. The study of almost 400,000 Medicare Advantage patients analyzed mortality within one year of completing the HCAHPS survey ${ }^{[31]}$. The mortality rate at one year was $3 \%$. There were no differences between satisfaction with overall care or physician communication between the groups. The only patient satisfaction measure found to be significantly different between the two groups was patient reports of getting care quickly. Patients who died within one year scored this measure 1.5 points higher than patients alive at one year $(p<.001)$. A significant linear trend in scores on timeliness of care in association with time to death was identified. Patients who died within 30 days provided higher scores on this measure than patients who died within 181-365 days $(p=.02)$. These results suggest that patients are not dissatisfied with their care at end of life, but were actually more satisfied with timeliness of care than patients who were alive at one year.

Complications and patient satisfaction were evaluated in colorectal surgery patients at a single institution by Gurland et al. ${ }^{[32]}$. The authors used the American College of Surgeons National Surgical Quality Improvement Program (ACS NSQIP) defined complications and evaluated for associations with all HCAHPS measures. The ACS NSQIP database is a nationally validated surgical quality improvement program. Complications that occur within 30 days of surgery are recorded by trained Surgical Clinical Reviewers (SCRs) and risk adjusted complication rates and nationally benchmarked data are reported back to hospitals. Patients with ACS NSQIP defined complications were less likely to provide the most 
positive survey response for the following questions: recommendation of hospital ( $p=.023)$, hospital staff responsiveness ( $p=.0003$ ), and quietness $(p=.002)$. Conversely, patients with complications were more satisfied with discharge information $(p=.042)$. Patients with medical complications were more satisfied with discharge information compared with patients who had technical complications $(p=.026)$.

Table 3. Patient outcomes and patient satisfaction

\begin{tabular}{|c|c|c|c|c|}
\hline Author & Journal & Patient Outcome & Satisfaction measure & Associations ${ }^{*}$ \\
\hline Elliott & $\begin{array}{l}\text { J Am Geriatr Soc } \\
2013\end{array}$ & End of life & $\begin{array}{l}\text { Plan } \\
\text { Prescription drug coverage } \\
\text { Physician } \\
\text { Specialists Care } \\
\text { Getting needed care } \\
\text { Getting care quickly } \\
\text { Physician communication } \\
\text { Medications } \\
\text { Information }\end{array}$ & Getting care quickly (-) \\
\hline Gurland & $\begin{array}{l}\text { J Am Coll Surg } \\
2013\end{array}$ & $\begin{array}{l}\text { Complications after } \\
\text { colectomy }\end{array}$ & $\begin{array}{l}\text { Overall satisfaction } \\
\text { Ranking of } 9 \text { or } 10 \\
\text { Recommendation of hospital } \\
\text { Physician communication } \\
\text { Nurse communication } \\
\text { Medication communication } \\
\text { Pain management } \\
\text { Clean room } \\
\text { Quiet room } \\
\text { Staff responsiveness } \\
\text { Discharge information }\end{array}$ & $\begin{array}{l}\text { Recommendation of hospital }(+) \\
\text { Staff responsiveness }(+) \\
\text { Quiet room }(+) \\
\text { Discharge information }(-)\end{array}$ \\
\hline Isaac & $\begin{array}{l}\text { Health Serv Res } \\
2010\end{array}$ & PSI & $\begin{array}{l}\text { Overall satisfaction } \\
\text { Ranking of } 9 \text { or } 10 \\
\text { Recommendation of hospital } \\
\text { Physician communication } \\
\text { Nurse communication } \\
\text { Medication communication } \\
\text { Pain management } \\
\text { Clean and quiet room } \\
\text { Staff responsiveness } \\
\text { Discharge information }\end{array}$ & $\begin{array}{l}\text { Decubitus ulcer }(+) \\
\text { Iatrogenic infection }(+) \\
\text { Respiratory failure }(+) \\
\text { Pulmonary embolism/deep vein } \\
\text { thrombosis }(+) \\
\text { Sepsis }(+) \\
\text { Hemorrhage }(+)\end{array}$ \\
\hline Jha & $\begin{array}{l}\text { N Engl J Med } \\
2009\end{array}$ & $\begin{array}{l}\text { Readmission } \\
\text { CHF } \\
\text { PN }\end{array}$ & Discharge information & $\begin{array}{l}\text { CHF readmission }(+) \\
\text { PN readmission }(+)\end{array}$ \\
\hline Boulding & $\begin{array}{l}\text { AJMC } \\
2011\end{array}$ & $\begin{array}{l}\text { Readmission } \\
\text { CHF } \\
\text { PN } \\
\text { AMI }\end{array}$ & $\begin{array}{l}\text { Overall satisfaction (averaged } \\
\text { ranking of } 9 \text { or } 10 \text { and } \\
\text { recommendation of hospital) } \\
\text { Discharge information }\end{array}$ & $\begin{array}{l}\text { Overall }(\mathrm{CHF}+, \mathrm{PN}+, \mathrm{AMI}+) \\
\text { Discharge }(\mathrm{CHF}+, \mathrm{PN}+)\end{array}$ \\
\hline
\end{tabular}

Note. ${ }^{*}(+)$ indicates a positive correlation between the survey domain and patient satisfaction, (-) indicates a negative correlation between the survey domain and patient satisfaction.

CHF: Congestive heart failure, PN: Pneumonia, AMI: Acute myocardial infarction, PSI: Patient Safety Indicators

Another measurement of complication rates are patient safety indicators (PSIs), which were developed by the AHRQ ${ }^{[33]}$. PSIs include in hospital complications and adverse events based on administrative data. A study of both medical and surgical PSIs ${ }^{[29]}$ found mixed correlations with all HCAHPS measures. Lower rates of decubitus ulcer PSIs were significantly associated with improved satisfaction on all HCAHPS measures $(p<.05)$. On the other hand, failure to rescue 
did not correlate with any HCAHPS domains. Iatrogenic infection was associated with lower satisfaction on 4 of 9 HCAHPS measures. Analysis of surgical PSIs demonstrated that low rates of respiratory failure and pulmonary embolism/deep venous thrombosis were most associated with high patient satisfaction (5 of 9 each). On the other hand, sepsis was only associated with 2 HCAHPS measures and hemorrhage was only associated with overall recommendation of the hospital. When evaluating medical and surgical HCAHPS scores by quartile, top quartile hospitals for cleanliness and quietness had fewer iatrogenic infections as compared with bottom quartile hospitals (1.8 events per 1,000 vs. 2.3 events per $1,000, p<.01)$. Hospitals scoring highest for staff responsiveness were found to have fewer decubitus ulcers as compared with lower scoring hospitals (20.7 events per 1,000 vs. 25.8 events per 1,000, $p<.01$ ). While results were mixed across PSIs and HCAHPS domains, there was a trend toward improved satisfaction with lower PSI rates.

CMS has emphasized the importance of hospital readmission as an outcome recently with legislature tying reimbursement to readmission rates. This is one dimension of patient care that has been shown to consistently correlate. Jha et al. ${ }^{[34]}$ found an association between higher patient satisfaction with the discharge process and lower readmission rates for both CHF and pneumonia. Patients with CHF in the highest satisfaction quartile were readmitted $22.4 \%$ of the time compared with $24.7 \%$ readmission rate in the lowest quartile ( $p<.001$ ). The same trend was noted for patients with pneumonia (17.5 vs. $19.5 \%, p<.001)$. Similar results were obtained by Boulding and colleagues ${ }^{[35]}$ who also found patient satisfaction with discharge planning was significantly associated with lower readmission rates in CHF patients $(p<.001)$ and pneumonia patients $(p=.02)$. Overall satisfaction was inversely associated with readmission in CHF, PN, and AMI patients. Readmissions are the only outcome found to reliably correlate with patients' perceptions of care in the current literature, although it is unknown whether these differences are clinically significant.

While patient outcomes were more reliably associated with patients' perceptions of care than quality and safety measures, these relationships were inconsistent across studies. In fact, patients with complications were more satisfied with discharge processes and patients who died within one year of the survey were more satisfied with timeliness of care.

\section{Discussion}

It is undeniable that patient satisfaction is an important measure of hospital care. It is clear that as we move into an era of pay for performance, we must strive to keep patients' needs at the center of our care. Therefore, tools that assess patients' perception of care will assure medical providers are attentive to the needs of the patients. However, it is important that we draw a distinction between patient centeredness of care and other quality of care measures given that patient satisfaction has not been shown to reliably predict traditional measures of quality and safety. We have undertaken this review to understand the relationships between patient centered care and traditional measures of hospital quality and safety. While there seems to be a trend between quality of care, patient safety, and patient outcomes correlating with patient satisfaction on the HCAHPS survey, the association has been inconsistent. Some studies have actually identified inverse relationships between these measures and patients' perceptions of care. More effort is needed to better characterize predictors of patient satisfaction and the association with quality and safety measures and patient outcomes.

While the HCAHPS survey has been studied extensively and it is the first national, publicly reported survey assessing patient satisfaction with health care, there are limitations to the survey and to the existing knowledge around interpretation of survey results. Studies have demonstrated acceptable hospital level reliability and internal consistency reliability for the survey after adjusting for patient case mix variables ${ }^{[5,12]}$. In addition to the variables included in the case mix adjustment, other factors have been assessed including diagnosis related group and time from discharge to survey completion and have not been found to influence response bias. While previous studies have identified patient variables, survey mode, and nonresponse biases, there are likely many other factors, which contribute to response bias. Similarly, little is known about disease specific variables and how they influence patient satisfaction. It is unlikely that a patient who is hospitalized for acute myocardial infarction and a patient who undergoes elective hernia repair have the same concerns, expectations, and goals for their hospital stay. Given that HCAHPS scores are now tied to Medicare reimbursement, it is imperative that 
other potential predictors of patient satisfaction are assessed to ensure that hospitals are being compared and reimbursed fairly.

Of the 10 studies reviewed here, no two studies evaluated the same data over the same time period, which makes comparison and interpretation of the results difficult. In addition, the level of analysis differed between studies with some evaluating survey results at the health plan level while others assessed patient satisfaction at the hospital or patient level. The number of hospitals included in the studies ranged from 1 to 2,583 and in most studies it was unclear which hospitals were evaluated. Although HCAHPS data is publicly available online, investigators are unable to obtain explanatory variables of interest for every hospital on the Hospital Compare website and therefore the number of included hospitals varies widely across studies.

Similarly, data in the studies summarized here was obtained from 2005-2013. The survey has changed over time and therefore the questions evaluated by the various studies differ, making comparisons difficult. It is unknown if there have been changes in what contributes to patients' perceptions of care over time. Since the HCAHPS survey has been implemented, transparency of results and dependence of hospital reimbursement on scores has motivated hospitals to improve patient satisfaction. It may be that hospitals have identified ways to improve patients' perceptions of care independent of quality and safety of care. This might explain differences in how satisfaction relates to quality and safety measures over time.

Comparisons between studies are also limited as the definition of the outcome of interest, patient satisfaction, varied across studies. Some studies evaluated all HCAHPS measures, while others focused on the overall satisfaction measures. Two studies developed summary composite HCAHPS measures, which averaged all measures ${ }^{[23,30]}$. In addition, some studies evaluated percentage of top HCAHPS scores and others divided top scores into quartiles. All HCAHPS scores were obtained from the Hospital Compare website and are already case mix adjusted, but some studies adjusted for other variables found to be associated with satisfaction scores, such as bed size, location, and hospital ownership ${ }^{[23,28]}$. Differing definitions of patient satisfaction further contribute to difficulty comparing and contrasting these studies.

The evaluation of patient outcomes in relation to satisfaction poses even more challenges. While a culture of safety and quality of care is likely to be relatively uniform across a single hospital, patient outcomes differ from patient to patient. When looking at patient outcomes, it is unknown if the patients who suffered poor outcomes were the same patients who completed the HCAHPS surveys. One exception was the end of life study by Elliott et al. ${ }^{[31]}$ which actually demonstrated improved satisfaction with getting care quickly in patients with one-year mortality. With other outcome measures, complications and readmissions, it is unknown if the patients completed the satisfaction surveys before or after the poor outcome. Given that most outcomes are recorded within 30 days of discharge and patients have six weeks to return HCAHPS surveys, it is likely that some patients completed the survey prior to their poor outcome while others completed the survey after the outcome of interest had occurred. For example, post-operative complications have been found to occur after discharge up to $40 \%$ of the time ${ }^{[36,37]}$. Although the majority of complications occur in the first two weeks after discharge ${ }^{[36]}$, it is impossible to know if patients completed HCAHPS surveys before or after complications and readmissions occurred. All of these issues likely contribute to the mixed results and difficulties interpreting the current literature.

Given all the limitations in published studies, it is difficult to know how closely related quality and safety measures and patient outcomes are with patient satisfaction on the HCAHPS survey. Further studies are needed to better assess these relationships. An ideal study would include a large number of patients across a wide variety of hospitals. Patient specific satisfaction and outcome data would be collected as well as concurrent hospital compliance with process measures and culture of safety surveys. The timing of survey completion is key to understanding how patient satisfaction correlates with post-discharge outcomes. Finally, patient interviews to identify why patients chose their answers would provide additional information about what factors are most important to individual patients. 


\section{Conclusion}

In summary, while patient satisfaction is a separate and equally important component of quality of care, it does not reliably correlate with traditional measures of quality and safety of patient care. Specifically, we found that while compliance with process measures tends to be associated with improved patient satisfaction, these relationships are inconsistent across studies. The literature assessing the relationship between culture of safety and patients' perceptions of care is limited with conflicting conclusions. In terms of patient outcomes, high readmission rates have been consistently correlated with poor patient satisfaction, while worse complication rates and mortality at one year are associated with higher patient satisfaction scores. Similar to how traditional markers of quality and safety have been examined, it will be important to identify variables that contribute to patient satisfaction in order to improve the patient experience and thereby improve satisfaction scores on the HCAHPS survey.

\section{References}

[1] HCAHPS. Fact Sheet. Available from: http://www.hcahpsonline.org/files/August\%202013\%20HCAHPS\%20Fact\%20Sheet2.pdf

[2] Goldstein E, Farquhar M, Crofton C, et al. Measuring hospital care from the patients' perspective: an overview of the CAHPS Hospital Survey development process. Health Serv Res. 2005; 40: 1977-1995. PMid: 16316434. http://dx.doi.org/10.1111/j.1475-6773.2005.00477.x

[3] Giordano LA, Elliott MN, Goldstein E, et al. Development, implementation, and public reporting of the HCAHPS survey. Med Care Res Rev. 2010; 67: 27-37. PMid: 19638641. http://dx.doi.org/10.1177/1077558709341065

[4] Levine RE, Fowler FJ, Jr., Brown JA. Role of cognitive testing in the development of the CAHPS Hospital Survey. Health Serv Res. 2005; 40: 2037-2056. PMid: 16316437. http://dx.doi.org/10.1111/j.1475-6773.2005.00472.x

[5] Keller S, O'Malley AJ, Hays RD, et al. Methods used to streamline the CAHPS Hospital Survey. Health Serv Res. 2005; 40: 2057-2077. PMid: 16316438. http://dx.doi.org/10.1111/j.1475-6773.2005.00478.x

[6] Elliott MN, Swartz R, Adams J, et al. Case-mix adjustment of the National CAHPS benchmarking data 1.0: a violation of model assumptions? Health Serv Res. 2001; 36: 555-573. PMid: 11482589.

[7] Fowler FJ, Jr., Gallagher PM, Nederend S. Comparing telephone and mail responses to the CAHPS survey instrument. Consumer Assessment of Health Plans Study. Med Care. 1999; 37: MS41-49. PMid: 10098558. http://dx.doi.org/10.1097/00005650-199903001-00005

[8] Zaslavsky AM, Zaborski LB, Cleary PD. Factors affecting response rates to the Consumer Assessment of Health Plans Study survey. Med Care. 2002; 40: 485-499. PMid: 12021675. http://dx.doi.org/10.1097/00005650-200206000-00006

[9] Elliott MN, Zaslavsky AM, Goldstein E, et al. Effects of survey mode, patient mix, and nonresponse on CAHPS hospital survey scores. Health Serv Res. 2009; 44: 501-518. PMid: 19317857. http://dx.doi.org/10.1111/j.1475-6773.2008.00914.x

[10] O'Malley AJ, Zaslavsky AM, Elliott MN, et al. Case-mix adjustment of the CAHPS Hospital Survey. Health Serv Res. 2005; 40: 2162-2181. PMid: 16316443. http://dx.doi.org/10.1111/j.1475-6773.2005.00470.x

[11] De Vries H, Elliott MN, Hepner KA, et al. Equivalence of mail and telephone responses to the CAHPS Hospital Survey. Health Serv Res. 2005; 40: 2120-2139. PMid: 16316441. http://dx.doi.org/10.1111/j.1475-6773.2005.00479.x

[12] Dyer N, Sorra JS, Smith SA, et al. Psychometric properties of the Consumer Assessment of Healthcare Providers and Systems (CAHPS(R)) Clinician and Group Adult Visit Survey. Med Care. 2012; 50 Suppl: S28-34. PMid: 23064274. http://dx.doi.org/10.1097/MLR.0b013e31826cbc0d

[13] Morales LS, Elliott MN, Weech-Maldonado R, et al. Differences in CAHPS adult survey reports and ratings by race and ethnicity: an analysis of the National CAHPS benchmarking data 1.0. Health Serv Res. 2001; 36: 595-617. PMid: 11482591.

[14] Petrullo K, Lamar S, Nwankwo-Otti O, et al. The Patient Satisfaction Survey: What does it mean to your bottom line? Journal of Hospital Administration. 2012; 2: 1-8. http://dx.doi.org/10.5430/jha.v2n2p1

[15] VanLare JM, Conway PH. Value-based purchasing--national programs to move from volume to value. N Engl J Med. 2012; 367: 292-295. PMid: 22830460. http://dx.doi.org/10.1056/NEJMp1204939

[16] Chassin MR. Improving the quality of health care: what's taking so long? Health Aff (Millwood). 2013; 32: 1761-1765. PMid: 24101066. http://dx.doi.org/10.1377/hlthaff.2013.0809

[17] Haynes AB, Weiser TG, Berry WR, et al. Changes in safety attitude and relationship to decreased postoperative morbidity and mortality following implementation of a checklist-based surgical safety intervention. BMJ Qual Saf. 2011; 20: 102-107. PMid: 21228082. http://dx.doi.org/10.1136/bmjqs.2009.040022 
[18] Singer S, Lin S, Falwell A, et al. Relationship of safety climate and safety performance in hospitals. Health Serv Res. 2009; 44: 399-421. PMid: 19178583. http://dx.doi.org/10.1111/j.1475-6773.2008.00918.x

[19] Randmaa M, Martensson G, Leo Swenne C, Engstrom M. SBAR improves communication and safety climate and decreases incident reports due to communication errors in an anaesthetic clinic: a prospective intervention study. BMJ Open. 2014; 4: e004268. PMid: 24448849. http://dx.doi.org/10.1136/bmjopen-2013-004268

[20] Sexton JB, Helmreich RL, Neilands TB, et al. The Safety Attitudes Questionnaire: psychometric properties, benchmarking data, and emerging research. BMC Health Serv Res. 2006; 6: 44. PMid: 16584553. http://dx.doi.org/10.1186/1472-6963-6-44

[21] Sorra JS, Dyer N. Multilevel psychometric properties of the AHRQ hospital survey on patient safety culture. BMC Health Serv Res. 2010; 10: 199. PMid: 20615247. http://dx.doi.org/10.1186/1472-6963-10-199

[22] Lyu H, Wick EC, Housman M, et al. Patient satisfaction as a possible indicator of quality surgical care. JAMA Surg. 2013; 148: 362-367. PMid: 23715968. http://dx.doi.org/10.1001/2013.jamasurg.270

[23] Sorra J, Khanna K, Dyer N, et al. Exploring relationships between patient safety culture and patients' assessments of hospital care. J Patient Saf. 2012; 8: 131-139. PMid: 22785344. http://dx.doi.org/10.1097/PTS.0b013e318258ca46

[24] Etchegaray JM, Thomas EJ. Comparing two safety culture surveys: safety attitudes questionnaire and hospital survey on patient safety. BMJ Qual Saf. 2012; 21: 490-498. PMid: 22495098. http://dx.doi.org/10.1136/bmjqs-2011-000449

[25] HEDIS. Available from: www.ncqa.org/HEDISQualityMeasurement

[26] Hospital Quality Alliance Process of Care Measures. Available from: http://www.cms.gov/Medicare/Quality-Initiatives-Patient-Assessment-Instruments/HospitalQualityInits/HospitalProcessOfCare Measures.html

[27] Schneider EC, Zaslavsky AM, Landon BE, et al. National quality monitoring of Medicare health plans: the relationship between enrollees' reports and the quality of clinical care. Med Care. 2001; 39: 1313-1325. PMid: 11717573. http://dx.doi.org/10.1097/00005650-200112000-00007

[28] Jha AK, Orav EJ, Zheng J, Epstein AM. Patients' perception of hospital care in the United States. N Engl J Med. 2008; 359: 1921-1931. PMid: 18971493. http://dx.doi.org/10.1056/NEJMsa0804116

[29] Isaac T, Zaslavsky AM, Cleary PD, Landon BE. The relationship between patients' perception of care and measures of hospital quality and safety. Health Serv Res. 2010; 45: 1024-1040. PMid: 20528990. http://dx.doi.org/10.1111/j.1475-6773.2010.01122.x

[30] Lehrman WG, Elliott MN, Goldstein E, et al. Characteristics of hospitals demonstrating superior performance in patient experience and clinical process measures of care. Med Care Res Rev. 2010; 67: 38-55. PMid: 19638640.

http://dx.doi.org/10.1177/1077558709341323

[31] Elliott MN, Haviland AM, Cleary PD, et al. Care experiences of managed care Medicare enrollees near the end of life. J Am Geriatr Soc. 2013; 61: 407-412. PMid: 23379270. http://dx.doi.org/10.1111/jgs.12121

[32] Gurland BH, Merlino J, Sobol T, et al. Surgical complications impact patient perception of hospital care. J Am Coll Surg. 2013; 217: 843-849. PMid: 24035448. http://dx.doi.org/10.1016/j.jamcollsurg.2013.06.015

[33] AHRQ. Patient Safety Indicators. Available from: www.qualityindicators.ahrq.gov/modules/psi_overview.aspx

[34] Jha AK, Orav EJ, Epstein AM. Public reporting of discharge planning and rates of readmissions. N Engl J Med. 2009; 361: 2637-2645. PMid: 20042755. http://dx.doi.org/10.1056/NEJMsa0904859

[35] Boulding W, Glickman SW, Manary MP, et al. Relationship between patient satisfaction with inpatient care and hospital readmission within 30 days. Am J Manag Care. 2011; 17: 41-48. PMid: 21348567.

[36] Kazaure HS, Roman SA, Sosa JA. Association of postdischarge complications with reoperation and mortality in general surgery. Arch Surg. 2012; 147: 1000-1007. PMid: 23165614. http://dx.doi.org/10.1001/2013.jamasurg.114

[37] Morris MS, Deierhoi RJ, Richman JS, et al. The Relationship Between Timing of Surgical Complications and Hospital Readmission. JAMA Surg. 2014. 